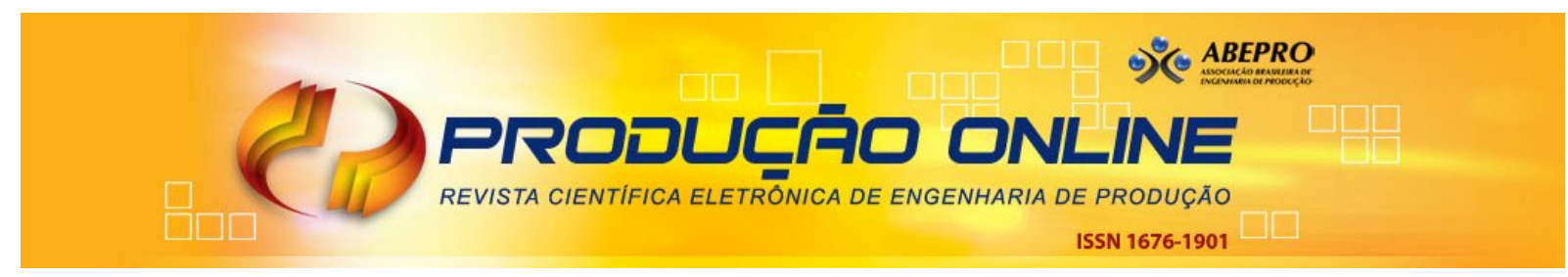

\title{
PROBLEMA DE ROTEAMENTO EM ARCOS CAPACITADO E PERIÓDICO APLICADO A UM CONTEXTO REAL
}

\section{PERIODIC CAPACITATED ARC ROUTING PROBLEM APPLIED IN A REAL CONTEXT}

\author{
Guilherme Vinicyus Batista* E-mail: guivbatista89@gmail.com \\ Cassius Tadeu Scarpin*E-mail: cassiusts@gmail.com \\ * Universidade Federal do Paraná, (UFPR), Curitiba, $\mathrm{PR}^{1}$
}

\begin{abstract}
Resumo: Um bom planejamento de inspeção e manutenção em ferrovias é essencial para garantir o fluxo dos trens e evitar possíveis acidentes. Essa inspeção deve ser feita periodicamente por veículos que transitam sobre os trilhos coletando dados e identificando falhas que precisam ser corrigidas. $\mathrm{O}$ objetivo desse artigo é apresentar uma modelagem matemática, baseada em programação linear binária, capaz de solucionar esse problema. Este problema é uma aplicação real do Problema de Roteamento em Arcos Capacitado e Periódico (PCARP). No PCARP cada um dos arcos de uma rede tem demandas periódicas ao longo de um horizonte de tempo bem definido, devendo ser criadas rotas para cada carro de modo que todas as necessidades sejam atendidas da melhor maneira possível sem exceder a capacidade dos carros. A aplicação proposta apresenta características diferenciadas das já apresentadas na literatura devido ao fato de não necessitar voltar a um depósito ao final de um dia e poder atrasar algum atendimento se necessário. O resultado obtido é satisfatório, atendendo as demandas com um movimento sincronizado dos veículos.
\end{abstract}

Palavras-chave: Problema de Roteamento em Arcos Capacitado e Periódico (PCARP), Ferrovias, Manutenção, Inspeção, Modelagem Matemática

\begin{abstract}
A good inspection and maintenance planning in railways is essential to ensure the flow of trains and avoid possible accidents. This inspection should be performed periodically by vehicle traveling on rails collecting data and identifying gaps that need to be corrected. The aim of this paper is to present a mathematical model based on binary linear programming, capable of solving this problem, which is a real application of Periodic Capacitated Arc Routing Problem (PCARP). In the PCARP each arc of a network has a demand over a well-defined time horizon and routes must be created for each car so that it covers all the requests in the best way possible without exceeding the vehicles capacity at service. The proposed application has different characteristics to those already proposed in the literature because the vehicle does not need to come back to the depot at the end of the day and the service can be delayed if necessary. The result is satisfactory, covering the demands with a synchronized movement of vehicles.
\end{abstract}

Keywords: Periodic Capacitated Arc Routing Problem (PCARP), Railways, Maintenance, Inspection, Mathematical Modelling

\section{INTRODUÇÃO}

1 Texto resultado de dissertação de mestrado no Programa de Pós-Graduação em Métodos Numéricos em Engenharia - PPGMNE-UFPR, Curitiba - PR, 2014 
Muitos pesquisadores têm resolvido problemas logísticos como localização de facilidades, gerenciamento de armazéns e roteamento de veículos. Esses problemas têm sido solucionados envolvendo tanto decisões estratégicas como táticas e operacionais, o que pode trazer um grande ganho para as empresas envolvidas (HASHEMI DOULABI; SEIFI, 2013).

Empresas que trabalham com ferrovias fazem manutenção preditiva (inspeção) e preventiva em seus trilhos, visto que toda malha ferroviária deve ser avaliada para garantir o fluxo dos veículos e prevenir acidentes, como o tombamento e descarrilamento de trens. Para isso, sofisticados equipamentos capazes de fazer medições específicas se deslocam ao longo da malha e devem estar em locais estratégicos para fazer seu trabalho. A malha é subdividida em trechos, onde cada trecho tem um intervalo de tempo pré-determinado no qual devem ocorrer inspeções (periodicidade). É importante definir uma rota para esses equipamentos de forma que as demandas de cada trecho sejam atendidas da melhor maneira possível, através de um planejamento de médio-longo prazo.

Problemas de roteamento de veículos, mais especificamente os problemas de roteamento em arcos, procuram resolver situações semelhantes. Nesse tipo de problema o principal objetivo é atender determinadas arestas que normalmente são relacionadas às ruas ou vias que fazem parte de uma rede. Entre os problemas que são tratados dessa forma encontram-se: coleta de lixo, entrega de correspondências, o despejamento de sal em vias com neve, manutenção em redes, entrega e coleta de produtos (CORBERÁN; PRINS, 2010).

O objetivo desse artigo é apresentar e aplicar uma modelagem matemática em programação linear binária capaz de solucionar uma adaptação de um problema real de inspeção de trilhos. Através da resolução do modelo matemático se obtêm uma solução onde as periodicidades de cada trecho são atendidas por uma quantidade de carros conhecida a priori. Cada um desses veículos deve percorrer uma rota de forma que ao longo de um horizonte de tempo pré-definido as demandas de cada via sejam satisfeitas da melhor forma possível.

Entre os problemas de roteamento em arcos encontra-se uma classe chamada de Problema de Roteamento em Arcos Capacitado e Periódico, do inglês Periodic Capacitated Arc Routing Problem (PCARP), no qual se encaixa o problema em questão. Um breve levantamento bibliográfico é apresentado na próxima seção apresentando trabalhos relevantes da literatura sobre o assunto. Na terceira seção Revista Produção Online, Florianópolis, SC, v.15, n. 3, p. 1080-1098, jul./set. 2015. 
um problema real é contextualizado, seguido pela formulação matemática e o resultado obtido nas seções 4 e 5 respectivamente, e finalmente tem-se as considerações finais em 6.

\section{PROBLEMAS DE ROTEAMENTO}

Os problemas de roteamento podem ser divididos em duas classes: roteamento em nós e roteamento em arcos. O roteamento em nós consiste em encontrar uma ou mais rotas através das quais um veículo visita alguns ou todos os nós de um grafo e o roteamento em arcos tem por objetivo determinar uma rota que passa por todos ou alguns arcos ou arestas de um grafo (MONROY; AMAYA; LANGEVIN, 2013).

Esses problemas se distinguem basicamente na modelagem em virtude da natureza dos serviços: passagens em arcos ou nós. Corberán e Prins (2010), trazem em seu trabalho um apanhado de resultados recentes encontrados na literatura sobre os Problemas de Roteamento em Arcos. Estes problemas tiveram origem no Problema das Pontes de Königsberg e tentam encontrar uma ou mais rotas que cubram todas (ou parcialmente) as ligações (arcos ou arestas) de um grafo, satisfazendo algumas restrições com o menor custo possível.

Konowalenko (2012) cita fatores listados na literatura que ampliam a diversidade e a complexidade dos problemas de roteamento: número de domicílios, tempo para servir um determinado nó ou arco, tamanho da frota, natureza da demanda, localização da demanda, restrições na capacidade dos veículos, natureza do grafo, entre outros.

\subsection{Problema de Roteamento em Arcos Capacitado}

Quando se trata de roteamento em arcos um dos problemas mais estudados na literatura é chamado em inglês pelo nome de Capacitated Arc Routing Problem (CARP), Problema de Roteamento em Arcos Capacitados. Ele é caracterizado por ter uma demanda não negativa associada a cada arco do grafo e é definido da seguinte forma: dado que cada veículo de uma frota tem uma determinada capacidade, esses veículos devem atravessar os arcos coletando ou entregando determinadas demandas sem exceder sua capacidade, problema este proposto em Revista Produção Online, Florianópolis, SC, v.15, n. 3, p. 1080-1098, jul./set. 2015. 
Golden e Wong (1981). Frequentemente essa capacidade é associada à carga máxima que um veículo consegue carregar, mas também pode ser vinculado à máxima capacidade de trabalho do carro ou ainda à distância percorrida (CORBERÁN; PRINS, 2010).

Eiselt et al. (1995) afirmaram na época, que o CARP é provavelmente o mais importante problema na área de roteamento em arcos. Existem diversas maneiras de solucionar o CARP, algumas delas são apresentadas em: Hertz, Laporte e Mittaz (2000) utilizando um algoritmo baseado em Busca Tabu; Beullens et al. (2003) baseando-se em guided local search; Belenguer e Benavent (2003) utilizando planos de corte; Lacomme, Prins e Sevaux (2006) a partir de Algoritmos Genéticos; e o trabalho de Longo, Aragão e Uchoa (2006) transformando o problema em um Problema de Roteamento de Veículos Capacitado.

Entre os problemas mais estudados envolvendo roteamento em arcos está a gestão de resíduos sólidos. Ghiani et al. (2014) afirmam que as técnicas de Pesquisa Operacional têm permitido alcançar significantes economias nos processos, assim como ajudar na recuperação dos resíduos com aplicações em partições de território, seleção de dias de coleta, composição da frota e roteamento e scheduling dos veículos coletores. Simonetto e Borestein (2004) apresentam um sistema de apoio à decisão que ajuda no planejamento operacional de coleta seletiva.

O CARP é amplamente utilizado para resolver o problema da coleta de lixo urbano como uma decisão de nível operacional, onde são definidas rotas diariamente para cada veículo a fim de atender as demandas de cada via. Porém, em alguns problemas é necessário tomar uma decisão de nível tático, que envolva um horizonte de tempo maior com múltiplos períodos, sujeita a restrições que envolvem determinadas frequências. Lacomme, Prins e Ramdane-Chérif (2002a) fazem esta definição apresentando o Problema de Roteamento em Arcos Capacitado e Periódico (PCARP), suas variantes e um Algoritmo Genético para resolvê-lo.

\subsection{Problema de Roteamento em Arcos Capacitado e Periódico}

De maneira geral o PCARP é uma extensão natural do CARP, mas ao invés do problema ser resolvido para apenas um dia ele é expandido para múltiplos Revista Produção Online, Florianópolis, SC, v.15, n. 3, p. 1080-1098, jul./set. 2015. 
períodos (MONROY; AMAYA; LANGEVIN, 2013). Resolver o PCARP implica em determinar simultaneamente as decisões em nível tático e operacional durante um horizonte de tempo.

Outro nome dado para o problema foi Problema de Planejamento em Roteamento em Arcos em Lacomme, Prins e Ramdane-Chérif (2002b) devido ao fato de se considerar diversos dias. Com isso, foi possível resolver simultaneamente os problemas de planejamento alocando CARPs diariamente. Lacomme, Prins e Ramdane-Chérif (2002a) fazem seu trabalho baseado na coleta de lixo urbano da cidade de Troyes na França e o resolvem com um Algoritmo Genético.

Alguns conceitos de problemas periódicos aparecem em Eglese (1994) com o roteamento de veículos responsáveis por não permitir que haja acúmulo de neve nas vias dos países com invernos rigorosos. Nesse problema, o autor fez um planejamento para apenas um dia, no qual algumas vias deveriam ser tratadas mais de uma vez no intervalo de poucas horas.

Christofides e Beasley (1984) levam em conta a importância do período no roteamento de veículos, visando reduzir os custos de distribuição ao longo de alguns dias. O problema é solucionado designando rotas para cada dia através da resolução de um problema de p-medianas e então resolvendo um Problema do Caixeiro Viajante. Cada cliente deve ser atendido mais de uma vez durante o horizonte de tempo em uma combinação de dias, sempre limitado pela capacidade dos carros.

Uma decisão importante a ser tomada antes de trabalhar com o problema é decidir como serão tratadas as restrições de periodicidades ou frequências de cada arco e isso não deixa de ser uma decisão estratégica a ser tomada por um especialista. Normalmente as demandas são flutuantes de acordo com os dias ou espaçadas em restrições de intervalos de tempo para executar cada serviço (LACOMME; PRINS; RAMDANE-CHÉRIF, 2005).

As restrições correspondentes à periodicidade podem assumir basicamente duas formas e devem estar disponíveis ao formular o problema. A primeira forma consiste em definir um mínimo espaço de tempo em que cada tarefa deve ser executada ou um espaço de tempo máximo entre dois serviços. Outra forma é utilizar uma combinação de dias em que pode ser executado o serviço, se o horizonte de tempo for de $n p$ dias pode haver um conjunto para cada tarefa ao qual são designados os dias em que o arco deve ser atendido. Por exemplo, se um arco Revista Produção Online, Florianópolis, SC, v.15, n. 3, p. 1080-1098, jul./set. 2015. 
necessita ser atendido todos os dias, esse conjunto é representada pelo conjunto (1, $2, \ldots, n p)$, ou se for apenas no primeiro e terceiro dia do horizonte de tempo, o conjunto seria formado pelo conjunto $(1,3)$ (LACOMME; PRINS; RANDAMECHÉRIF, 2002).

A forma da combinação de dias é utilizada por Chu, Labadi e Prins (2005) e também por Lacomme, Prins e Ramdane-Chérif (2002) e é defendida pelos autores por existirem duas grandes vantagens: as restrições de espaço são satisfeitas implicitamente e os cálculos de demanda podem ser feitos antecipadamente em alguns casos.

$\mathrm{Na}$ prática o custo de servir um arco depende da demanda. Baseado nisso Lacomme e Prins (2005) propõe uma classificação para o PCARP dividindo inicialmente em duas classes A e B, que podem ser encontradas na Figura 1.

A CLASSE A representa o conjunto dos problemas em que a demanda não resulta de acumulação diária de algum fator, como em muitos serviços de prevenção, inspeção e monitoramento. Por exemplo, na pulverização de herbicidas em trilhos de trem: o tempo gasto por metro percorrido e a demanda ao longo do tempo é fixa.

Já a CLASSE B envolve problemas como a coleta de lixo, onde a demanda pela coleta aumenta conforme vão passando os dias. Além disso, podem ser subdivididos em problemas acíclicos (subclasse B1) ou cíclicos (subclasse B2). Em B1, o horizonte de tempo é bem definido e a demanda é flutuante ao longo do tempo, as vezes é necessário passar muitas vezes e em outros casos não, como no caso da remoção de plantas em rodovias, visto que no inverno as plantas não crescem. Em B2 o intervalo de tempo se repete continuamente, por exemplo, a coleta de lixo que se repete de semana em semana. 
Figura 1 - Uma simples classificação do PCARP

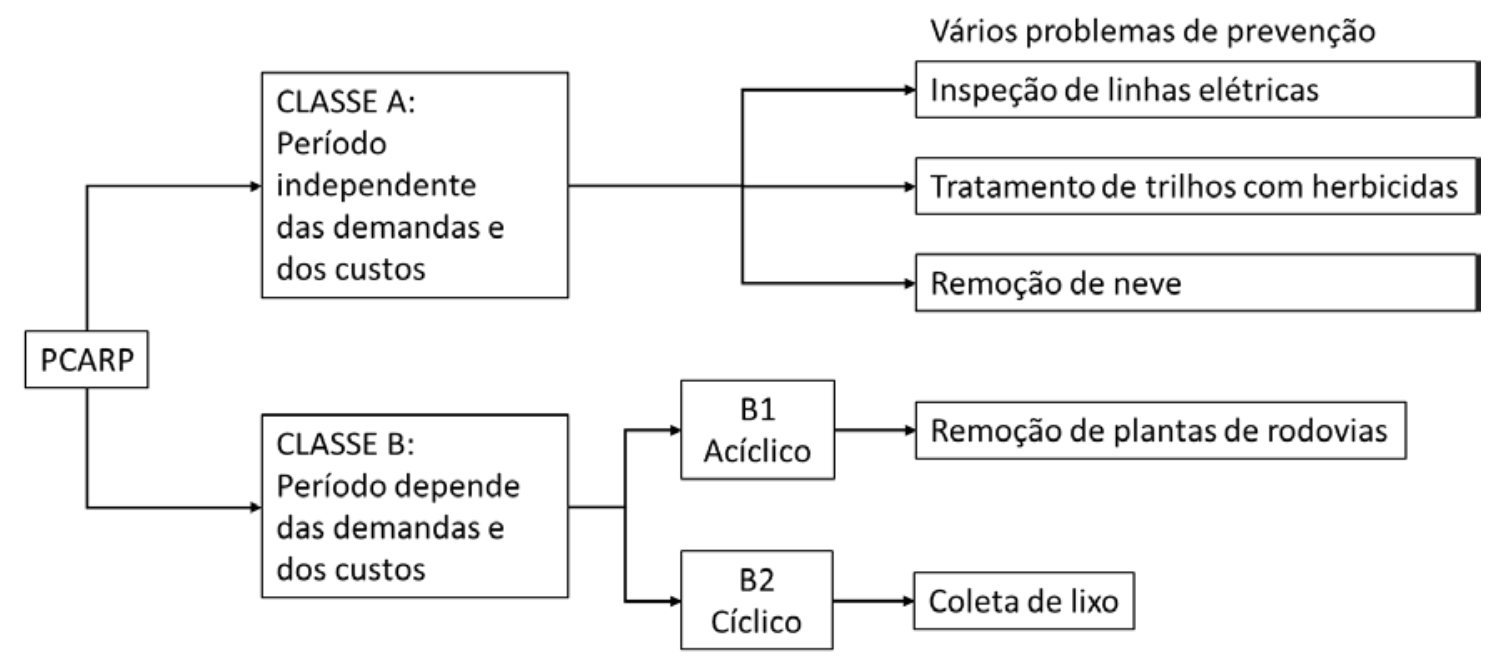

Fonte: Lacomme, Prins e Ramdane-Chérif (2005)

Encontram-se na literatura três modelos matemáticos de alta relevância que tratam do PCARP. Os dois primeiros são propostos por Chu, Labadi e Prins (2004, 2005) e o outro proposto por Monroy, Amaya e Langevin (2013). Chu, Labadi, \& Prins (2004) foram os primeiros a propor uma modelagem matemática para 0 PCARP onde também foi proposto lower bounds prelimares. Eles conseguiram resultados ótimos em softwares comerciais apenas para instâncias muito pequenas com 5 períodos e 10 arcos, fato já esperado devido à natureza do problema - NPHard.

Chu, Labadi e Prins (2006) aplicam dois algoritmos para resolver o PCARP. O primeiro é uma Heurística de Melhor Inserção e o segundo é chamado Scatter Search baseado em busca local. Ainda lembram que uma solução factível deve ter suas necessidades atendidas o número de vezes que forem necessárias (frequências), cada viagem deve iniciar e terminar no depósito, cada tarefa deve ser designada no máximo uma vez em um dia e ainda a capacidade do veículo deve ser respeitada.

Chen et al. (2009) trabalham com múltiplos períodos utilizando um conceito de Problema de Roteamento em Arcos em Múltiplos dias, do inglês Multiday Arc Routing Problem (MARP). Ele agrupa os problemas de roteamento aqui citados numa categoria de Problemas de Roteamento em Arcos Determinísticos e introduz os conceitos de Problema de Roteamento em Arcos Probabilístico e MARP para resolver um problema no contexto de entrega de pequenos pacotes. Em ambos os 
problemas, considera-se uma probabilidade de atravessar determinado arco em algum período.

Kansou e Yassine (2009) combinaram um algoritmo da colônia de formigas com uma heurística de inserção para resolver o PCARP, conseguindo resultados robustos e com uma performance rápida. Cada serviço podia acontecer em uma combinação de dias que satisfizessem as frequências, este problema foi chamado de Problema de Roteamento em Arcos Capacitado e Periódico Misto em virtude da natureza do grafo.

Lacomme, Prins e Ramdane-Chérif (2002b) tem um PCARP com dois objetivos, minimizar a frota de veículos e minimizar os custos das viagens. Para resolver este problema utilizaram um Algoritmo Genético híbrido.

Monroy, Amaya e Langevin, (2013) trabalham com o PCARP em um contexto de monitoramento da malha rodoviária que é realizado periodicamente. As ruas ou rodovias são divididas em classes de acordo com a necessidade de vigilância e a hierarquia das vias. Cada categoria tem sua demanda (número de passagens) durante um horizonte de tempo já definido. O objetivo é designar um conjunto de rotas satisfazendo as frequências de cada classe de vias em cada sub período de tempo sem exceder a capacidade do veículo.

\section{APLICAÇÃO}

Uma das aplicações do PCARP está relacionada ao serviço de manutenção, o qual se configura como um investimento, de extrema importância para uma empresa, que proporciona uma redução não somente nos custos de reparo de equipamentos como no custo de paradas do processo (MARCORIN; LIMA, 2003).

A aplicação real do problema que será aqui tratado está associada aos conceitos de manutenção preditiva e preventiva. Marcorin e Lima (2003) definem manutenção preventiva como um conjunto de ações que visam prevenir a quebra e está associado à intervenções periódicas geralmente programadas segundo uma frequência. Já a manutenção preditiva caracteriza-se pela medição e análise de variáveis da máquina que possam prognosticar uma eventual falha, com isso as equipes de manutenção podem se programar para evitar gastos desnecessários.

A malha ferroviária brasileira tem grande extensão e os equipamentos disponíveis para analisar e garantir o perfeito estado dos trilhos devem ser Revista Produção Online, Florianópolis, SC, v.15, n. 3, p. 1080-1098, jul./set. 2015. 
colocados em locais estratégicos para fazer seu trabalho de manutenção preditiva. Um caso bem específico é um trem de pequeno porte que mede o vão entre trilhos. Esse trem, que deve percorrer toda a malha, é um equipamento que necessita de um alto investimento. Com a possibilidade de locomoção desse pequeno trem sobre os trilhos, faz-se necessário determinar diariamente onde ele deve executar seu trabalho. Porém, existem algumas restrições quanto a sua capacidade diária de trabalho e os trechos que deve percorrer.

Alguns trechos com alto fluxo de trens necessitam de medições com uma frequência maior, ou seja, é necessário executar esse serviço em intervalos de tempo mais curtos do que em outros. Essa frequência, necessária em cada arco, está vinculada a periodicidade de cada trecho. A Figura 2 representa uma simplificação de um problema real.

$\mathrm{Na}$ representação nota-se que há 5 tipos de periodicidades diferentes: o trecho de maior frequência tem a necessidade de uma passagem a cada 10 dias, os demais tem necessidade de uma passagem a cada 15 dias, 20 dias, 30 dias e, finalmente, 60 dias para os trechos com menor necessidade. Exemplificando, o arco 19-20 representa um trecho que tem uma periodicidade de 10 dias, ou seja, a cada 10 dias esse arco deve ser atravessado ao menos uma vez, já o arco 10-11 uma vez a cada 30 dias.

O mapa pode ser representado por um grafo, e como o Brasil é um país de dimensões continentais nele existem diversas cidades que são atravessadas pelas arestas e seriam candidatas a pontos de parada. Um problema tão grande como o representado pela Figura 2 ao ser modelado exigiria uma grande quantidade de variáveis e restrições. Por isso foi feita a simplificação ilustrada que resultou em uma redução do horizonte de tempo e das periodicidades de cada trecho, somadas a um agrupamento de pontos e arestas. 


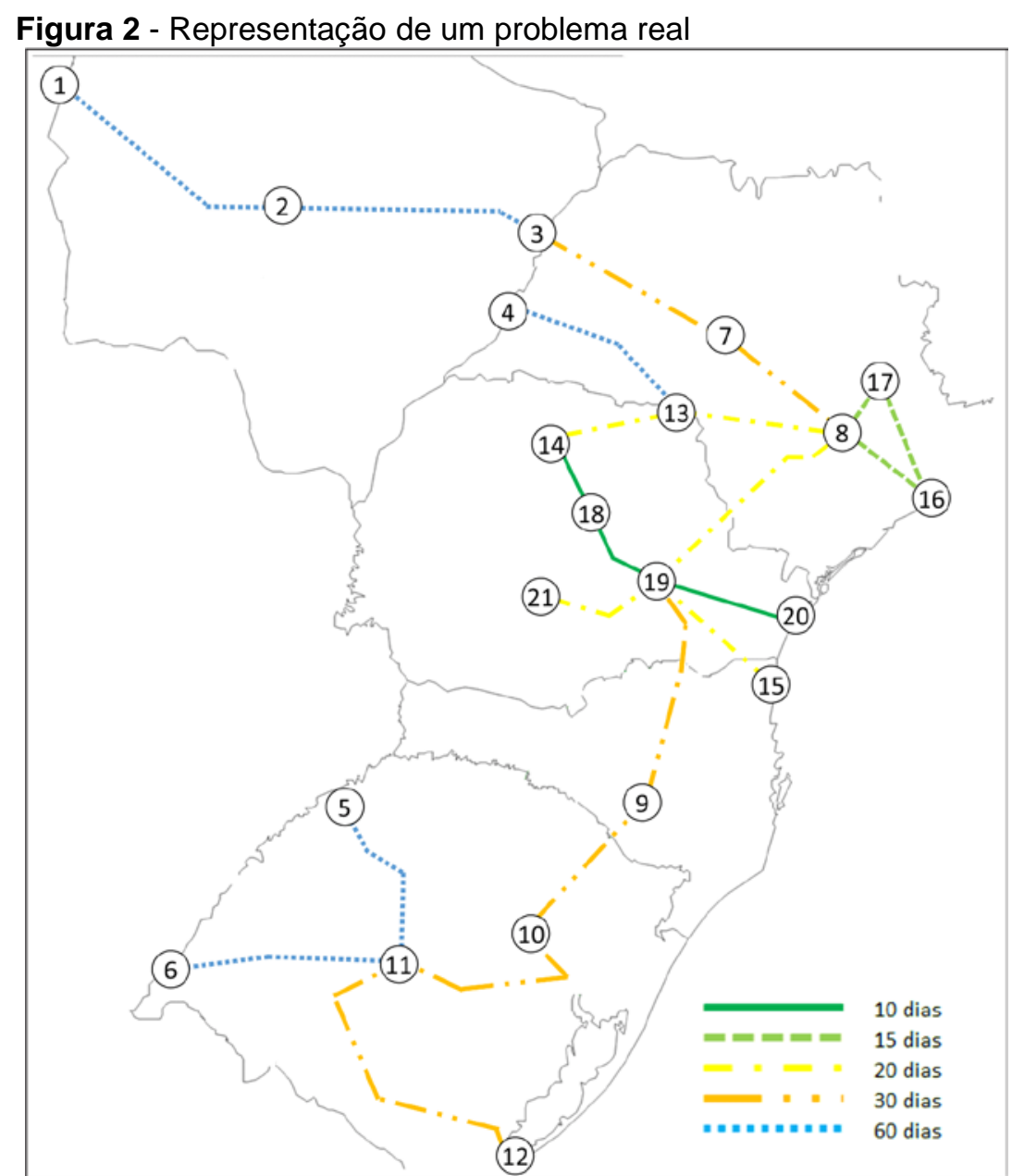

Fonte: O autor

São disponibilizados dois trens, denominados a partir desse momento como carro ou veículo, para atender os arcos. Estes têm sua capacidade limitada à quilometragem que conseguem percorrer diariamente em cada trecho. Vale ressaltar que em alguns trechos os deslocamentos são mais lentos e em outros mais rápidos. De maneira geral, o deslocamento de um ponto até outro representa o movimento que um carro consegue fazer em um período (um dia no caso).

Uma particularidade desse problema é que os equipamentos que se deslocam ao longo de toda a malha não necessitam voltar a um ponto específico ao final do dia (também conhecido como depósito). O deslocamento de cada equipamento, em geral, é lento e, durante o intervalo de um dia, o trem inicia seus trabalhos em um local e termina em outro, a partir do qual se deve iniciar o trabalho no próximo dia.

Em cada um dos pontos enumerados de 1 até 21 os operadores e os equipamentos podem pernoitar, dado que o trabalho não é executado 24 horas por 
dia. Analisando a Figura 2 ainda tem-se a informação de que durante um dia de serviço é possível fazer o deslocamento do ponto 19 para o 20, ou do 1 para o 2 e jamais fazer um deslocamento do ponto 1 ao ponto 3 em um único dia.

O horizonte de tempo é definido baseado no arco de maior periodicidade. No caso do exemplo da Figura 2 temos um horizonte de tempo de 60 dias. Ou seja, dentro do período de 60 dias os arcos devem ter sua demanda atendida. Isso implica que os arcos com periodicidade de 10 dias devem ter seis passagens durante esses 60 dias, de forma que a cada 10 dias o trecho seja percorrido ao menos uma vez.

Como é um problema cíclico, ao final dos 60 dias o carro deve estar no mesmo ponto onde iniciou seu trajeto no dia 1, o que equivale a dizer que o que deve ocorrer no dia 61 é exatamente o que ocorre no primeiro dia. Arcos com menor periodicidade devem levar em conta a sequência dos dias após o final dos primeiros 60 dias.

\section{MODELAGEM MATEMÁTICA}

Para resolver o problema é proposta uma formulação que parte de um grafo não direcionado $G=(X, E)$ com $n$ pontos, logo tem-se o conjunto $X=\left\{x_{1}, x_{2}, \ldots, x_{n}\right\}$, e $m$ arestas em $E=\left\{e_{1}, e_{2}, \ldots, e_{m}\right\}$ que devem ser percorridos por $n k$ carros definidos pelo conjunto $K=\{1,2, \ldots, k\}$. Cada arco e é formado por um par de nós, e é chamado de $x_{i j}=\left(x_{i}, x_{j}\right)$ ou $[i, j]$ e associado a um custo $c_{i j}$.

Eglese (1994) considerou a capacidade dos veículos em uma restrição que levava em conta a máxima distância que poderia ser feita durante uma rota. Para simplificar o problema aqui tratado a capacidade de cada carro também foi convertida na máxima distância que um carro pode se deslocar, ou seja, 1 arco por período.

A demanda ou periodicidade de cada arco é expressa na quantidade máxima de períodos em que o arco deve ser atendido ao menos uma vez $M P\left(x_{i j}\right)$. Tem-se ainda o horizonte de tempo $H$, formado por $n p$ períodos, onde cada período é simbolizado pela letra $p$.

O modelo é baseado em Programação Linear Binária, logo para a resolução do problema foram criadas três variáveis binárias apresentadas a seguir: 


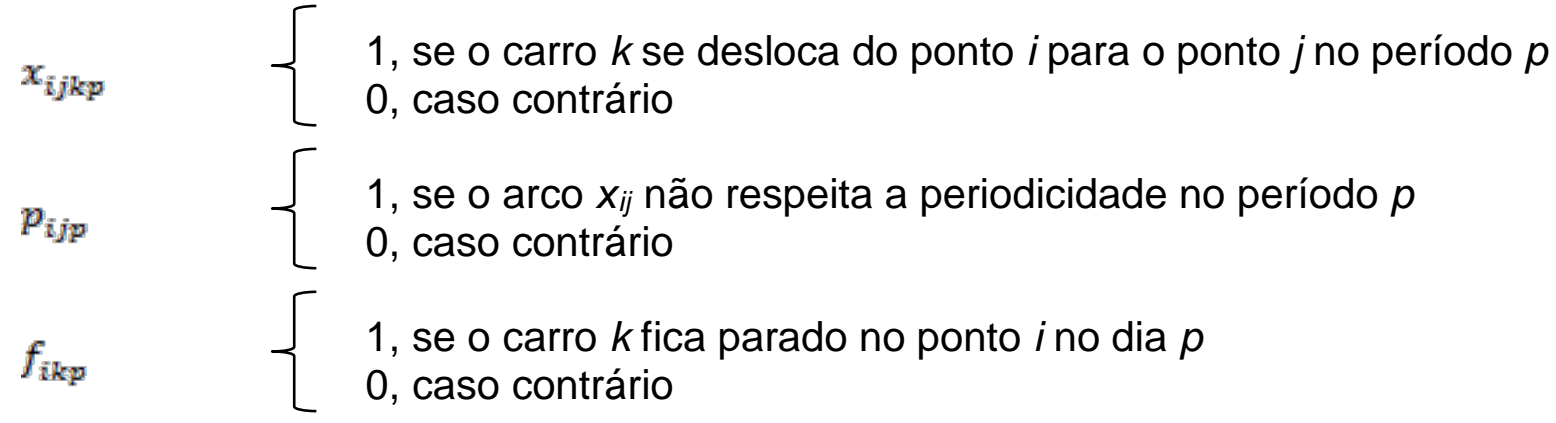

As variáveis $p_{i j p}$ e $f_{i k p}$ foram criadas para que o problema tenha uma viabilidade. Cada variável $p_{i j p}$ tem associado a ela um custo $P U_{i j}$, que é uma punição caso a periodicidade de um arco não seja atendida. Isso permite que na modelagem proposta haja um atraso no atendimento de alguns arcos, visto que a quantidade deve ser a menor possível.

A variável $f_{i k p}$ permite que o carro folgue em um determinado dia, pois algumas vezes é possível obter uma solução com menos deslocamentos se o carro esperar para atravessar um arco. Ou simplesmente quando essa variável assumir valor 1, pode significar que as periodicidades podem ser atendidas em menor tempo, ou seja, o horizonte de tempo pode diminuir.

A função objetivo procura minimizar os custos de deslocamento e o número de atrasos, sendo dada pela equação (1):

$\min Z=\sum_{[i, j] \in E} \sum_{k=1}^{n k} \sum_{p=1}^{n p} c_{i j} * x_{i j k p}+\sum_{[i, j] \in E} \sum_{E}^{n p} P U_{i j} * p_{i j p}$

O modelo está sujeitos às seguintes restrições:

$$
\begin{aligned}
& \sum_{[i, j] \in E} x_{i j k p}+f_{i k p}-\sum_{[i, j] \in E} x_{j i k, p+1}-f_{i k, p+1}=0 \quad \forall i \in X, k, p \\
& \sum_{[i, j] \in E} x_{i j k p}+\sum_{[i, j] \in E} x_{j i k p}+\sum_{i=1}^{n} f_{i k p}=1 \quad \forall p, k \\
& \sum_{k=1}^{n k}\left(\begin{array}{c}
x_{i j k p}+x_{j i k p}+x_{i j k, p+1}+x_{j i k, p+1}+\ldots \\
+x_{i j k, p+M P\left(x_{i j}\right)-1}+x_{j i k, p+M P\left(x_{i j}\right)-1}
\end{array}\right)+p_{i j p} \geq 1 \quad \forall[i, j] \in E, p \\
& x_{i j k p}, p_{i j p}, f_{i k p} \quad \in\{0,1\} \quad \forall[i, j] \in E, k, p
\end{aligned}
$$

A restrição (2) garante o fluxo diário dos carros permitindo a folga, sendo que, como serão formados ciclos, ao fim do período $n p$ o carro deve retornar para o mesmo local em que estava no dia 1 , ou seja, o período $n p+1$ é igual ao dia 1 , e Revista Produção Online, Florianópolis, SC, v.15, n. 3, p. 1080-1098, jul./set. 2015. 
assim por diante. Isso vale para todo o horizonte de tempo e para todas as restrições.

Nos problemas reais podem ocorrer imprevistos e outros problemas que atrasem 0 atendimento a alguns arcos. A possiblidade de um carro folgar na modelagem permite que quando ocorra algum problema dessa natureza a folga compense o tempo perdido.

A restrição (3) garante que todos os carros tenham alguma designação para o dia $p$, além disso, garante implicitamente que a capacidade de cada carro não seja excedida. Tem-se basicamente que em cada dia cada veículo deve executar um movimento, podendo deslocar-se de $i$ para $j$, ou de $j$ para $i$, ou simplesmente ficar parado em um ponto $i$.

Já a restrição (4) é a mais complexa. Ela se refere à periodicidade de cada trecho que deve ser atendida. Na restrição (4) é possível que as variáveis $p_{i j p}$ assumam valor 1, ou seja, um arco pode ser atrasado em um dia ao custo de ser punido com um custo $P U_{i j}$. Contudo, se a necessidade for de apenas uma passagem durante todo o horizonte de tempo, a restrição pode ser simplificada para apenas um dia $p, p=1$, o que abrange todo o horizonte de tempo e a variável $p_{i j p}$ pode ser descartada, como feito para a aplicação aqui apresentada.

Em (5) as variáveis binárias são dimensionadas.

\section{RESULTADO}

Em testes computacionais do modelo apresentado nota-se que ao ser aplicado a diversas estruturas de grafos a memória do computador se esgota conforme a complexidade do problema e a solução obtida pode não ser de agrado. A fim de obter uma boa solução para o problema ilustrado o tempo para resolução do problema foi limitado a 24 horas no software CPLEX 12.4 em um computador com processador Intel Core i5, com processamento de $3,1 \mathrm{GHz}$ com $8 \mathrm{~Gb}$ de memória RAM em um sistema operacional de 64 bits.

A solução obtida será representada a partir da Figura 3 até a Figura 5, cada figura tem representadas as rotas dos dois carros para 20 dias de execução dividida em duas ilustrações de 10 dias cada. Foi escolhida a representação de 10 dias por ser a menor periodicidade a ser atendida. 
Figura 3 - Ilustração das rotas obtida para os dias de 1 a 20

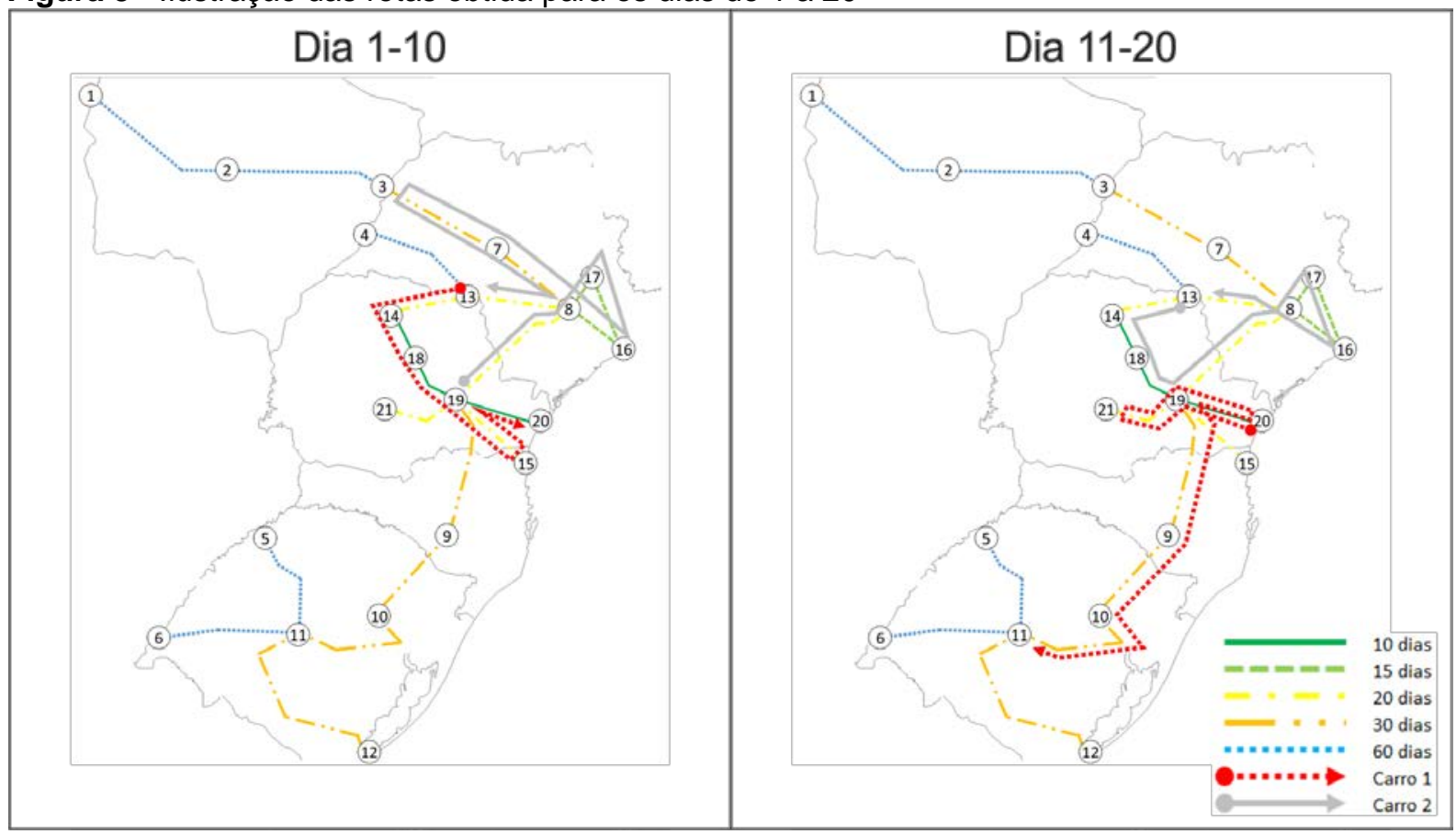

Fonte: $\mathrm{O}$ autor

Em algumas das ilustrações será possível notar que existem menos de 10 deslocamentos ao longo do horizonte de tempo, o que significa que houve folgas.

$\mathrm{Na}$ Figura 3 temos representados os movimentos que os carros devem fazer entre o dia 1 e o dia 10. A solução encontrada foi a seguinte:

- Carro 1: 13 - 14 - 18 - 19 - 15 - 19 - 20 - 20 - 20 - 20 - 20;

- Carro 2: 19 - 8 - 17 - 16 - 8 - 8 - 7 - 3 - 7 - 8 - 13 .

O que significa que o Carro 1 ficou parado no ponto 20 nos dias 7, 8, 9, 10, e o Carro 2 no dia 5 ficou parado no ponto 8.

Ainda na Figura 3 estão representados os movimentos entre os dias 11 e 20, os quais foram:

- Carro 1: 20 - 19 - 19 - 19 - 21 - 19 - 20 - 19 - 9 - 10 - 11;

- Carro 2: 13 - 14 - 18 - 19 - 8 - 17 - 16 - 16 - 16 - 8 - 13.

O Carro 1 ficou parado nos dias 12 e 13 no ponto 19, e o Carro 2 no ponto 16 nos dias 17, 18.

Nota-se que a maior parte das necessidades estão localizadas na região central do mapa, representados pelos estados do Paraná e São Paulo e as demais regiões tem menos necessidades de passagens. Como são dois carros, o que se espera como resultado é que enquanto um esteja cuidando da região mais "crítica" 
(com maior número de necessidades), outro carro se desloque para atender as regiões mais afastadas. Enquanto o Carro $1 \mathrm{em}$ vermelho se deslocou para o sul do mapa, o Carro 2 em cinza manteve sua rota na região central.

Na figura 4 os movimentos entre os dias 21 e 30 foram:

- Carro 1: 11 - 12 - 11 - 5 - 11 - 10 - 9 - 19 - 8 - 17 - 16;

- Carro 2: 13 - $14-18-19-15-19-19-20-20-19-18$.

O Carro 2 ficou parado nos pontos 19 e 20 nos dias 26 e 28 respectivamente. Analisando graficamente parece que o Carro 1 se deslocou muito mais do que o Carro 2, de fato é verdade, mas não apenas por causa das duas folgas do Carro 2. Vale lembrar que os deslocamentos são feitos baseados na duração de tempo para se deslocar de um ponto ao outro e não na sua distância. A distância interfere, porém o tempo tem uma relação direta com a velocidade em que cada trecho pode ser servido, isso faz com que em algumas regiões com tráfego menor de veículos e que necessitam de menos manutenção preditiva sejam atravessados mais rápido que outros.

Figura 4 - Ilustração das rotas obtida para os dias de 21 a 40

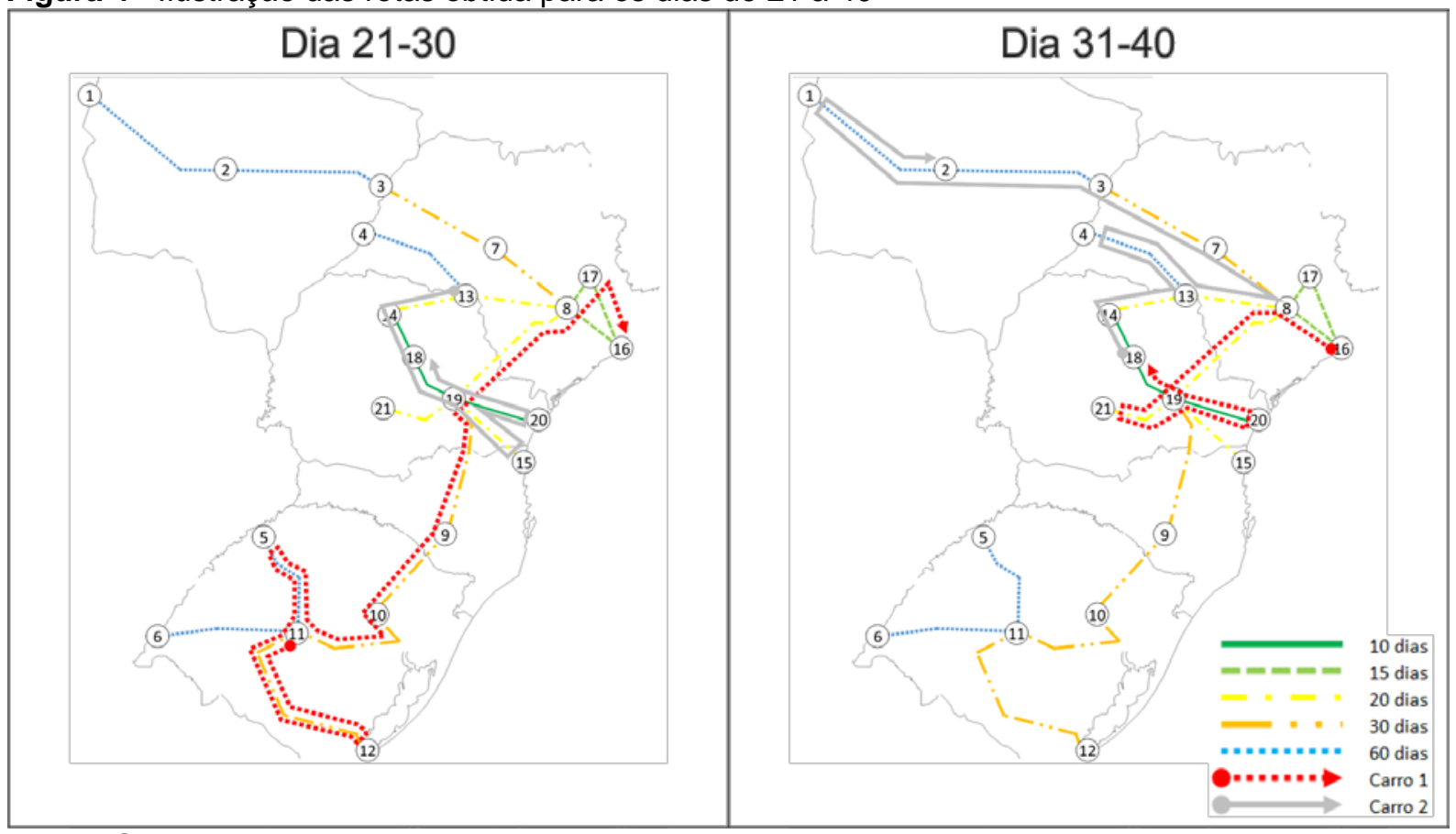

Fonte: O autor

Ainda na Figura 4 foram finalizados os serviços dos 30 primeiros dias, metade do horizonte de tempo, e iniciou-se a segunda metade dos serviços, representados pelos dias 31 e 40 com os movimentos: 
- Carro 1: 16 - 8 - 19 - 21 - 21 - 21 - 21 - 19 - 20 - 19 - 18;

- Carro 2: 18 - 14 - 13 - 4 - 13 - 8 - 7 - 3 - 2 - 1 - 2.

O Carro 1 ficou parado no ponto 21 entre os dias 34 a 36. Como é o início da segunda metade das avaliações nos trilhos, os arcos com periodicidade de 30 devem começar a ser analisados, ao menos, pela segunda vez. Isto é o que de fato acontece, pois por exemplo, o arco 3-7 que foi medido logo no início da rota, tem novamente medições feitas no início dessa segunda metade do horizonte de tempo.

Os movimentos entre os dias 41 e 50 representados na Figura 5 são:

- Carro 1: 18 - 14 - 13 - 8 - 17 - 16 - 8 - 19 - 20 - 19 - 18;

- Carro 2: 2 - 3 - 7 - 8 - 19 - 15 - 15 - 15 - 19 - 9 - 10.

Onde o Carro 2 ficou parado no ponto 15 nos dias 46, 47. Novamente percebe-se que um carro fica concentrado na região centralizada enquanto outro atravessa o mapa indo aos pontos com menor necessidade.

A Figura 5 também apresenta os últimos 10 movimentos dos carros, realizados entre os dias 51 e 60:

Figura 5 - Ilustração das rotas obtida para os dias de 41 a 60

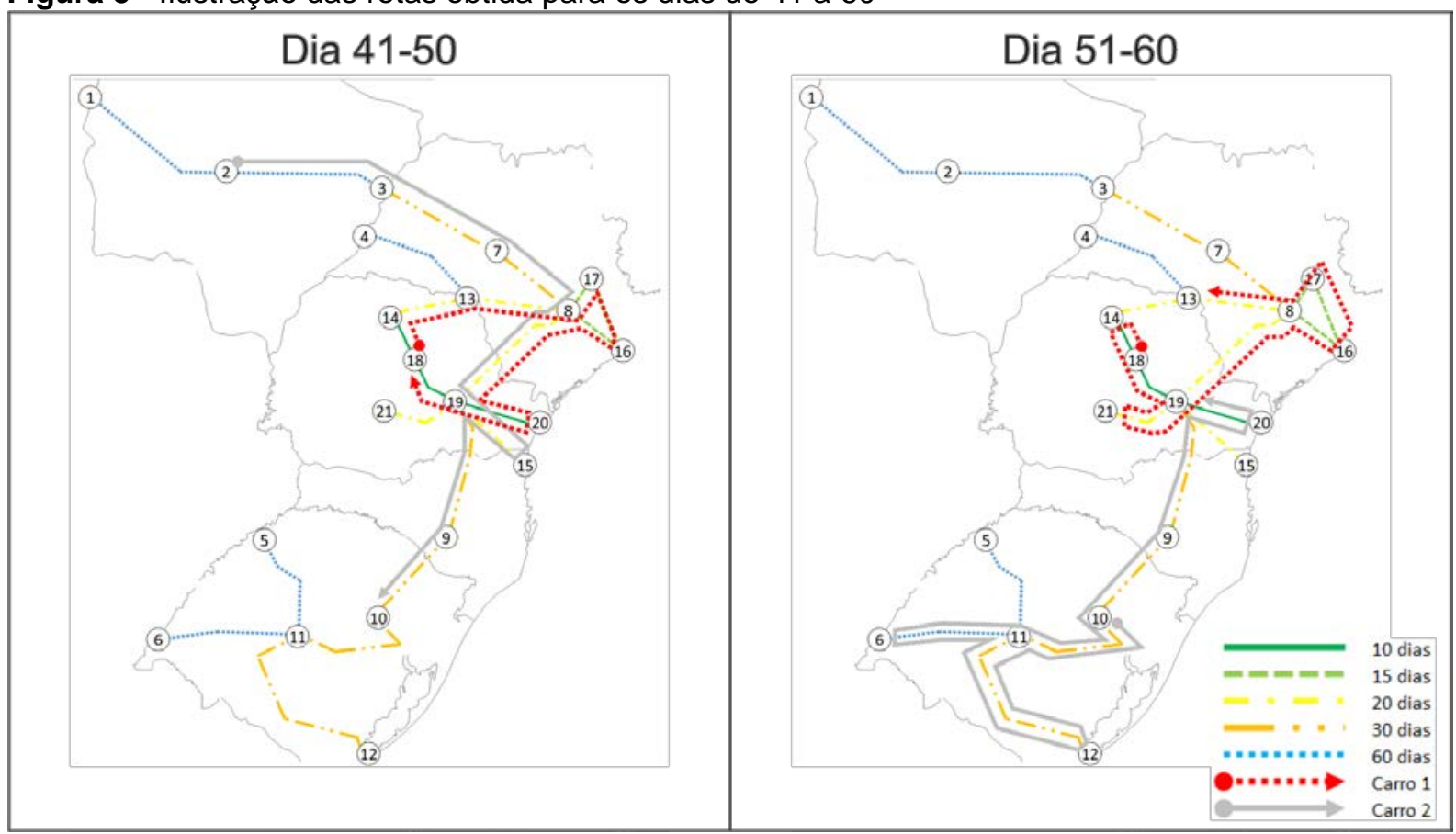

Fonte: O autor

- Carro 1: 18 - 14 - 18 - 19 - 21 - 19 - 8 - 16 - 17 - 8 - 13;

- Carro 2: 10 - 11 - 12 - 11 - 6 - 11 - 10 - 9 - 19 - 20 - 19. 
O Carro 1 iniciou seu trajeto e finalizou no ponto 13 e o mesmo acontece com o Carro 2 no ponto 19. Ao longo de todos os trajetos é possível perceber que todas a periodicidades são aceitas.

Os arcos em verde, que tem a maior periodicidade, são atravessados em todas as figuras e os arcos em azul apenas uma vez. Nota-se que os arcos em azul são bem intercalados no horizonte de tempo, por exemplo, na região sul existem basicamente dois arcos em azul, e como a região é visitada duas vezes, cada vez que um dos carros se desloca para lá um dos arcos é visitado.

Ao todo o carro 1 folgou 9 vezes e o carro 2 folgou 7 vezes. Essas folgas são extremamente importantes no problema real, pois podem ocorrer eventuais paradas nas máquinas por falha ou manutenção e as folgas acabariam compensando essas paradas.

\section{CONSIDERAÇÕES FINAIS}

Os problemas de roteamento em arcos têm grande aplicabilidade e podem ser adaptados conforme as necessidades. Abrangem um campo de atuação ainda não tão explorado como os problemas de roteamento por nós. Entre os problemas reais que podem ser resolvidos dessa forma encontram-se: coleta de lixo, manutenção, monitoramento e prevenção de rodovias, ferrovias, linhas elétricas, remoção de neve de ruas, entre outros.

O modelo proposto somado a simplificação do problema real permitiu que o problema apresentasse uma solução muito boa em que a segurança da via é mantida através da manutenção preditiva. É muito relevante fazer um planejamento de serviços que não se restrinjam a apenas um dia e abranjam um horizonte de tempo mais longo, pois assim é possível planejar bem todos os movimentos a serem tomados a fim de evitar que ocorram problemas e permitir que seja feito um "balé" sincronizado dos veículos para atender todas as necessidades conhecidas a priori. Deste modo, planejar visando um longo horizonte de tempo é, muitas vezes, necessário para manter um sistema estável e sem falhas.

O alto tempo de processamento é compensado pelo fato de se estar planejando para uma grande quantidade de dias que se repetirão formando ciclos, 24 horas de execução não são relevantes quando se trata de um planejamento que será feito para meses. Para trabalhos futuros pode-se desenvolver heurísticas e Revista Produção Online, Florianópolis, SC, v.15, n. 3, p. 1080-1098, jul./set. 2015. 
comparar soluções para escolher um método apropriado para resolução do problema.

\section{REFERÊNCIAS}

BELENGUER, J.; BENAVENT, E. A cutting plane algorithm for the capacitated arc routing problem. Computers \& Operations Research, v. 30, p. 705-728, 2003.

http://dx.doi.org/10.1016/S0305-0548(02)00046-1

BEULLENS, P.; MUYLDERMANS, L.; CATTRYSSE, D.; VAN OUDHEUSDEN, D. A guided local search heuristic for the capacitated arc routing problem. European Journal of Operational Research, v. 147, n. 3, p. 629-643, 2003. http://dx.doi.org/10.1016/S03772217(02)00334-X

CHEN, S.; GOLDEN, B.; WONG, R.; ZHONG, H. Arc-Routing Models for Small-Package Local Routing. Transportation Science, v. 43, n. 1, p. 43-55, 2009.

http://dx.doi.org/10.1287/trsc.1080.0255

CHRISTOFIDES, N.; BEASLEY, J. E. The Period Routing Problem. Networks, v. 14, p. 237-256, 1984. http://dx.doi.org/10.1002/net.3230140205

CHU, F.; LABADI, N.; PRINS, C. The Periodic Capacitated Arc Routing Problem Linear Programming Model, Metaheuristic and Lower Bounds. Journal of Systems Science and Systems Engineering, v. 13, n. 4, p. 423-435, 2004. http://dx.doi.org/10.1007/s11518-006$\underline{0174-y}$

CHU, F.; LABADI, N.; PRINS, C. Heuristics for the periodic capacitated arc routing problem. Journal of Intelligent Manufacturing, v. 16, n. 2, p. 243-251, 2005.

http://dx.doi.org/10.1007/s10845-004-5892-8

CHU, F.; LABADI, N.; PRINS, C. A Scatter Search for the periodic capacitated arc routing problem. European Journal of Operational Research, v. 169, p. 586-605, 2006.

http://dx.doi.org/10.1016/j.ejor.2004.08.017

CORBERÁN, A.; PRINS, C. Recent Results on Arc Routing Problems : An Annotated Bibliography. Networks, p. 50-69, 2010. http://dx.doi.org/10.1002/net.20347

EGLESE, R. W. Routeing winter gritting vehicles. Discrete Applied Mathematics, v. 48, n. 3, p. 231-244, 1994. http://dx.doi.org/10.1016/0166-218X(92)00003-5

EISELT, H. A.; GENDREAU, M.; LAPORTE, G. Arc Routing Problems , Part II : The Rural Postman Problem. Operations Research, v. 43, n. 3, p. 399-414, 1995a.

http://dx.doi.org/10.1287/opre.43.3.399

GOLDEN, B. L.; WONG, R. T. Capacitated Arc Routing Problem. Networks, v. 11, p. 305315, 1981. http://dx.doi.org/10.1002/net.3230110308

GHIANI, G.; LAGANÀ, D.; MANNI, E.; MUSMANNO, R.; VIGO, D. Operations research in solid waste management: A survey of strategic and tactical issues. Computers \&

Operations Research, v. 44, p. 22-32, 2014. http://dx.doi.org/10.1016/j.cor.2013.10.006 
HERTZ, A.; LAPORTE, G.; MITTAZ, M. A Tabu Search heuristic for the Capacitated Arc Routing Problem. Operations Research, v. 48, n. 1, p. 129-135, 2000.

http://dx.doi.org/10.1287/opre.48.1.129.12455

KANSOU, A.; YASSINE, A. Ant Colony System for the Periodic Capacitated Arc Routing Problem Problem. Proc. 2009 International Network Optimization Conference. Anais... p.17, 2009.

KONOWALENKO, F. Problema do Carteiro Chinês Não-Orientado e Misto para a Otimização de Rotas na Cidade de Irati / PR. 2012. 108 f. Dissertação de Mestrado PPGMNE. Universidade Federal do Paraná. Curitiba, 2012.

LACOMME, P.; PRINS, C.; RAMDANE-CHÉRIF, W. Evolutionary Algorithms for Multiperiod Arc Routing Problems. In: IPMU 2002 (Ed.); 9th Int. Conf. on Information Processing and Management of Uncertainty in Knowledge-Based systems. Anais... p.1-8, 2002a.

Annecy, France: ESIA-University of Savoie.

LACOMME, P.; PRINS, C.; RAMDANE-CHÉRIF, W. Planning Problems in Arc Routing. In: F. U.-E. de Valencia (Ed.); Actes de PMS 2002 (EURO Working Group on Project

Management and Scheduling). Anais... p.232-235, 2002b. Valencia, Espanha.

LACOMME, P.; PRINS, C.; RAMDANE-CHÉRIF, W. Evolutionary algorithms for periodic arc routing problems. European Journal of Operational Research, v. 165, p. 535-553, 2005. http://dx.doi.org/10.1016/j.ejor.2004.04.021

LACOMME, P.; PRINS, C.; SEVAUX, M. A genetic algorithm for a bi-objective capacitated arc routing problem. Computers \& Operations Research, v. 33, n. 12, p. 3473-3493, 2006. http://dx.doi.org/10.1016/j.cor.2005.02.017

LONGO, H.; DE ARAGÃO, M. P.; UCHOA, E. Solving capacitated arc routing problems using a transformation to the CVRP. Computers \& Operations Research, v. 33, n. 6, p. 1823-1837, 2006. http://dx.doi.org/10.1016/j.cor.2004.11.020

MARCORIN, W. R.; LIMA, C. R. L. Análise dos Custos de Manutenção e de Nãomanutenção de Equipamentos Produtivos. Revista de Ciência \& Tecnologia, v. 11, n. 22, p. 35-42, 2003.

MONROY, I. M.; AMAYA, C. A.; LANGEVIN, A. The Periodic Capacitated Arc Routing Problem with Irregular Services. Discrete Applied Mathematics, v. 161, p. 691-701, 2013. http://dx.doi.org/10.1016/i.dam.2011.05.014

SIMONETTO, E. O.; BORENSTEIN, D. SCOLDSS - Sistema de Apoio à Decisão Aplicado ao Planejamento e Distribuição da Coleta Seletiva de Resíduos Sólidos. Revista Produção Online, Florianópolis-SC, v. 4, n.4, p. 1-8, 2004. http://dx.doi.org/10.14488/1676-

1901.v4i4.1857

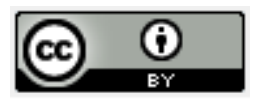

Artigo recebido em 22/12/2014 e aceito para publicação em 12/02/2015 DOI: http://dx.doi.org/ 10.14488/1676-1901.v15i3.1962 